\title{
Influence des efforts induits par le fluide sur la technologie des robinets
}

\section{The influence of fluid-induced stress on valves design}

\author{
A. Boissier \\ Direction des Etudes et Recherches, EDF, Les Renardières
}

\section{Introduction}

Compte tenu de l'importance du programme engagé en matière d'aménagement des centrales nucléaires, le Département Technologie des composants des Etudes et Recherches de l'EDF est amené à effectuer de nombreux essais dans le domaine de la robinetterie. Une part importante de cette activité consiste à faire manœuvrer les robinets d'une manière répétitive sur des boucles en eau ou vapeur dans des conditions voisines des conditions de service.

Cette activité englobe également l'étude des plans, la réception en usine, les essais statiques et l'expertise après les essais de manœuvre sur boucle.

A chaque incident, l'analyse des causes amène EDF à inciter le robinetier à effectuer certaines modifications, l'appareil est ensuite à nouveau essayé. On peut ainsi juger de l'efficacité des modifications apportées et au fil des années d'essai, il est possible de juger de l'évolution de la technologie en général, et de celle de tel type de robinet en particulier.

L'exemple traité ici est un cas d'école créé à partir de l'expérience acquise sur plusieurs matériels de même type et fonctionnant dans des conditions voisines. Il rassemble en une seule présentation une évolution technologique qui est en fait le fruit d'une expérience portant sur plusieurs années.

\section{Présentation du problème}

Soit à interrompre (ou à permettre) un débit d'eau dans une tuyauterie de diamètre interne $75 \mathrm{~mm}$ et de $7 \mathrm{~mm}$ d'épaisseur. Les conditions sont les suivantes :

Pression : 170 bar

Température : $285^{\circ} \mathrm{C}$

Débit : $30 \mathrm{~m}^{3} / \mathrm{h}$

Différence de pression : 150 bar
Temps de mancuvre : 8 secondes

Nature du corps : Z6 CND 17-12 (AISI 316)

On suppose, de plus, que le fluide reste monophasique, ce qui exclut les phénomènes de cavitation.

\section{Présentation de l'organe obturant}

Il s'agit d'un robinet vanne à sièges obliques et à obturateur en forme de coin monobloc élastique représenté sur les planches 1 et 2 .

L'étanchéité au sectionnement est obtenue par l'action du coin empêché de remonter par la tige et dont l'élasticité permet de maintenir une pression de contact des faces de l'opercule contre les sièges. Les portées d'étanchéité sont revêtues de stellite.

Le mouvement de l'opcrculc est guidé par des barrettes soudées sur le corps, l'opercule possède des oreilles qui glissent le long des barrettes. En principe, ce n'est qu'en fin de fermeture (et en début d'ouverture), qu'il y a frottement des portées d'étanchéité les unes sur les autres, le guidage étant précisément prévu pour empêcher ce frottement pendant les mancuvres.

N.B. Seule l'étanchéité des faces aval est fonctionnellement suffisante; en fait, les deux faces participent à l'étanchéité, mais ce sont les portées aval qui, du fait du fluide, subissent les pressions de contact les plus élevées. La différence de pression se traduit en position fermée par une force totale sur l'opercule d'environ 6000 daN. La pression de contact des surfaces au niveau des portées d'étanchéité est alors de 400 bar environ. $A$ l'ouverture et à $\Delta P$ maintenu, les efforts sont repris par les guidages. La pression de contact au niveau des oreilles d'opercule est d'environ 1200 bar. 


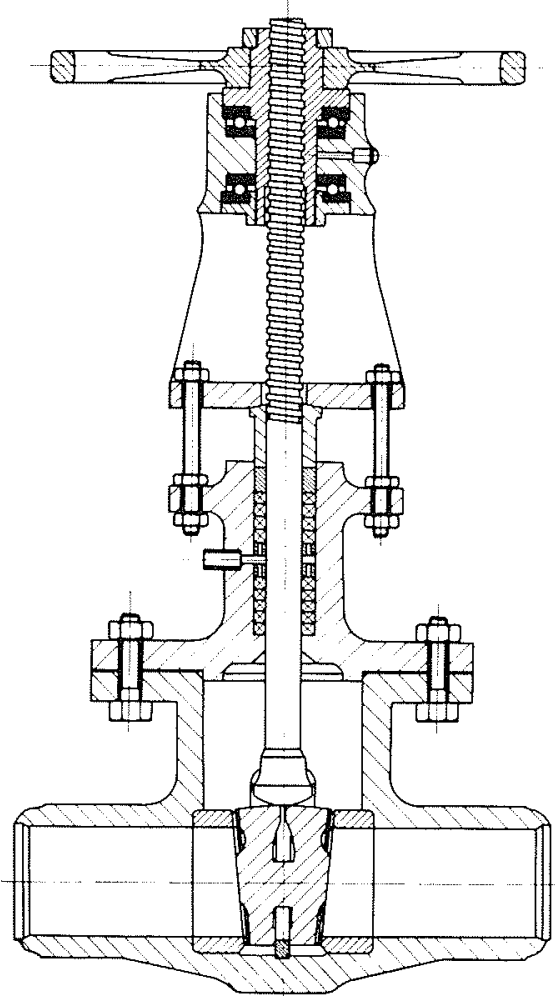

Planche 1 - Vanne à sièges obliques et à coins flexibles.

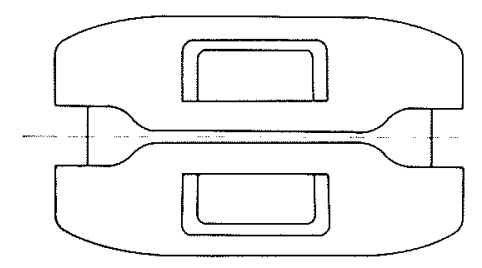

OPERCULE - VUE DE DESSUS

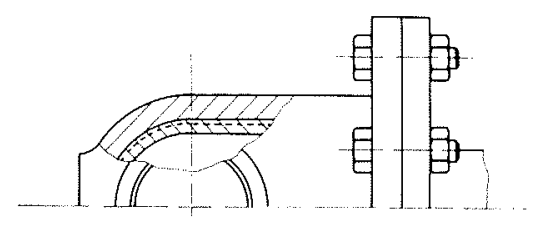

Vue de gauche - coupe partielle

Planche 2 - Vanne à sièges obliques et à coins flexibles.

\section{Incidents et modifications apportées}

On va maintenant passer en revue tous les incidents survenus sur ce type de matériel en rappelant toutefois que ce n'est pas forcément le même robinet qui a connu tous les incidents, et que certains d'entre eux présentés ici séparément, ont pu se produire en fait simultanément, le cloisonnement qui est fait est seulement attaché à la relation cause-effet.

\section{Incident $n^{\circ} l$}

Nature : Manœuvre incomplète. Le limiteur de couple déclenche la motorisation avant la fermeture complète. Constat : Grippage des guidages.

Cause: Guides d'opercule de même nature que la barrette de guidage.

Remède : En effectuant un dépôt de stellite sur les oreilles d'opercule, on améliore les conditions de frottement du guidage d'opercule.

\section{Incident $n^{\circ} 2$}

Nature : Après démontage et remontage, il n'y a plus étanchéité au sectionnement.

Constat : L'opercule ne s'adapte pas parfaitement sur ses sièges, on s'aperçoit en faisant un retournement de $180^{\circ}$ de l'opercule, que l'application devient bonne. Cause : Il n'y a pas symétrie de l'ensemble operculesièges-guide.

Remède 1 : Repérage du sens de montage de l'opercule ; cette solution présente des inconvénients évidents pour la maintenance, pratiquement en effet, cela amène à avoir pour chaque appareil un opercule spécialement adapté pour rechange; de plus, le repérage n'est pas un remè de absolu contre un mauvais montage.

Remède 2 : Changement complet du processus de réalisation et de montage restituant la symétrie et permettant l'interchangeabilité.

\section{Incident $n^{\circ} 3$}

Nature: dito incident $\mathrm{n}^{\circ} 1$.

Constat : Grippage des guidages, marquage des portées d'étanchéité

Cause : A chaque manœuvre, en particulier à la fermeture, il y a une légère rotation de l'opercule qui vient heurter sur les guides et sur les portées d'étanchéité. Remède : Pose sur la tige d'un bras anti-rotation, entrấnant une modification du dessin de l'arcade.

\section{Incident $n^{\circ} 4$}

Nature: dito incident $n^{\circ} 1$.

Constat : Grippage des portées d'étanchéité. Détérioration des guides.

Causes : Jeux aux guides trop importants, le guidage en fin de fermeture est effectué par les portées d'étanchéité et non par les guides.

Remède : Réduction des jeux. 


\section{Incident $n^{\circ} 5$}

Nature : Refus de fermeture après plusieurs mancuvres. Constat : Opercule coincé sur les guides, détérioration des guides d'opercule et du corps. Il y a eu déformation permanente de l'opercule qui a eu tendance à "se fermer".

Cause : Les oreilles d'opercule en se rapprochant sont venues emprisonner les barrettes, ce qui est à l'origine du coincement.

L'opercule est sous-dimensionné par rapport à la motorisation qui a été calculée pour appliquer un couple de serrage nécessaire à l'étanchéité. En fait, comme l'ont prouvé les essais faits à cette occasion, le couple nécessaire à l'étanchéité a été surévalué et, c'est en définitive, la motorisation qui est surdimensionnée par rapport à l'opercule.

Remède: Mettre une motorisation moins puissante, juste nécessaire pour obtenir l'étanchéité, ou diminuer la vitesse de manœuvre afin de diminuer les effets d'inertie, c'est l'étude des conditions de service qui doit orienter le choix.

\section{Améliorations possibles}

Face aux incidents qu'on vient d'examiner, on peut, outre les modifications apportées, imaginer encore des améliorations, en particulier au niveau du guidage de l'opercule.

On peut allonger les oreilles d'opercule et diminuer ainsi les pressions de contact au niveau des guidages.

Les conditions de frottement peuvent être également améliorées, en particulier entre guides d'opercule et barrettes de guidage ; il convient pour cela de bien choisir la nature des matériaux des portées en présence.
On peut également améliorer la technologie de mise en cuvre des barrettes de guidage qui pourraient être usinées soigneusement et mises en place par encastrement dans les corps. Cette dernière disposition devrait améliorer le guidage et assurer une meilleure symétrie de l'équipage mobile.

On s'est contenté de passer en revue les incidents qui interviennent au niveau du sectionnement. Des études analogues appuyées par des actions de recherche appropriées sont également menées pour l'étanchéité vers l'extérieur, tant à la jonction corps-chapeau qu'au presse-garniture. Ces études montrent qu'il est possible, surtout au presse-garniture, d'améliorer le comportement des robinets.

De même, la nécessité de réduire au maximum l'activité des circuits liés au circuit primaire des centrales nucléaires, conduit à rechercher des revêtements durs pour les surfaces frottantes, exempts de cobalt (les stellites contiennent environ $60 \%$ de cobalt).

\section{Conclusion}

L'appareil qui a évolué au fur et à mesure que s'approfondissait la connaissance des phénomènes liés à l'écoulement et à la technologie, est finalement assez différent du moins sous l'aspect fonctionnel, du produit de départ. Il s'agit là d'un bon exemple des améliorations que peut apporter l'expérience des essais ; il souligne l'intérêt qu'il y a de disposer de moyens d'essais capables de soumettre les robinets aux efforts réels, mais aussi, il met en évidence la nécessité de prendre en compte les efforts au niveau de la conception et d'en étudier leur incidence sur les surfaces frottantes.

\section{Discussion}

Président : M. R. CARDINAL

Sur proposition du Président, la discussion qui suit portait à la fois sur le présent mémoire et sur celui précédent de $M$. LEPHILIBERT.

Le Président remercie M. LEPHILIBERT et M. BOISSIER et ouvre la discussion sur les deux communications.

M. BONNIN, - Je voudrais poser deux questions à M. LEPHILIBERT :

Dans quelle mesure un meilleur tracé hydraulique de la chambre d'un robinet à soupape, en améliorant la répartition des pressions. peut-il améliorer ou annuler l'effort perpendiculaire sur la tige ?

Quelle peut-être l'influence des divers degrés de liberté nécessaires entre la tige et le clapet, sur les vibrations des robinets à soupape sous écoulement, en particulier aux ouvertures partielles?
M. LEPHILIBERT. - Voici deux réalisations concernant un robinet à tige montante non tournante.

Dans la première, une chambre permet d'égaliser à peu près les pressions autour du clapet. Malgré tout, nous avons eu des problèmes de grippage de la tige, ce qui prouve que cette utilisation n'était qu'approximative. Dans la deuxième réalisation, les effets sur la tige sont totalement annulés car le clapet, guidé dans un fourreau, est totalement indépendant de la tige dans le sens radial. Je pense avoir répondu à votre première question.

Pour la seconde, je suis beaucoup moins à l'aise, parce que nous ne connaissons pas bien les problèmes de vibrations; nous les subissons, mais nous ne les connaissons pas bien.

M. BONNIN. - Je parlais de la distribution de pression dans la chambre.

M. LEPHILIBERT. - En fait, cette chambre-là est plus importante que ne le nécessiterait le passage du clapet. Elle a 
été déterminée à la suite d'essais ; pourtant, dans les cas difficiles, je préconise la séparation des fonctions comme je l'a montrée dans les précédents dessins. En ce qui concerne les jeux, il est toujours possible de faire des prouesses, par exemple de faire fonctionner un robinet à tige tournante à $300 \mathrm{~b}$. Mais il faudra calibrer les jeux et les répartir entre les pièces de façon telle qu'on réunira d'excellents prototypes, mais que des déboires apparaîtront en production de séries. L'expérience prouve qu'à partir de $100 \mathrm{~b}$ il n'est pas possible de faire fonctionner statistiquement des robinets à tige tournante, ceci pour des raisons de contrôle qui devient trop poussé et d'échelonnement de jeux absolument inmaitrisable dans des fabrications de séries.

En ce qui concerne les vibrations des clapets, nous ne connaissons pas ce problème dans nos applications PWR : je ne peux donc pas répondre à cette question.

M. GROTLOH. - M. GROTLOH intervient pour donner quelques précisions sur les dispositions constructives adoptées - très faibles tolérances, guidages. . . - pour réduire autant que possible les vibrations.

M. HALLE. - Je pose une question à M. LEPHILIBERT.

A côté de l'étanchéité interne, vous savez que la majeure partie des incidents entrainant l'indisponibilité de tranches ont leur origine dans l'inétanchéité externe (fuite au presse garniture) Or on sait actuellement faire des presse-garnitures capables de 35000 mancuvres avant apparition de la première goutte d'eau, moyennant un certain nombre de précautions touchant la nature des garnitures et leur mise en œuvre. Nous constatons qu'à l'heure actuelle peu de constructeurs proposent des dispositifs convenables. Pourquoi ? Ne sont-ils pas convaincus ? En sont-ils dissuadés par la pression des acheteurs au niveau des prix ?

M. LEPHILIBERT. - En fait, les constructeurs sont obligés d'opérer par générations d'appareils.

En ce qui concerne l'étanchéité des presse-garnitures, la compréhension du phénomène a beaucoup évolué au cours des deux dernières années. On sait faire, actuellement, un pressegarnitures idéal compte tenu de l'état de la technologie. On ne savait pas le faire il $\mathrm{y}$ a six ans, au moment où les premières commandes du contrat-programme EDF ont été passées.

Voici ce que nous considérons comme le presse-garnitures idéal. Il comporte

- un système de guidage parfait de la tige ; celle-ci étant affranchie des efforts hydrauliques subis par le clapet ;

- un serrage élastique des tresses :

- un système d'éjection rapide des tresses.

Ce presse-garnitures est beaucoup plus performant que celui des premières générations de robinets, nous en sommes convaincus. Ma réponse est donc la suivante : les robinetiers savent faire, actuellement, des presse-garnitures performants ; il faut les introduire dans de nouvelles générations de robinets à l'occasion de nouveaux contracts.

M. AFLALO. - Je voudrais savoir si l'on a rencontré des problèmes consécutifs à des chocs thermiques et s'il y a eu des modifications de conception proposées aux constructeurs.

$M$. BOISSIER, - L'incident le plus fréquent que l'on a rencontré et qui soit đ'une manière certaine consécutif aux chocs thermiques, est une ou plusieurs fissurations des revêtements durs en stellite.

Le cas s'est produit sur une vanne à sièges parallèles. L'analyse a montré qu'il s'agissait d'un défaut de procédure de dépôt. Un autre incident concernait un robinet à soupape dont le revêtement en stellite s'est fendu radialement au cours des chocs thermiques. Un autre incident du même type, enfin, concernait un battant de clapet de non retour.
M. AFLALO. - Dans le même ordre d'idées, avez-vous fait des essais en fatigue thermique, consistant à appliquer des cycles de montée et de descente en température en nombre suffisant.

M. BOISSIER. - Nous n'effectuons pas d'essais de fatigue thermique, mais on soumet l'appareil à une série de 10 chocs thermiques. Il ne s'agit donc pas d'endurance, cependant la tenue $d u$ choc thermique est bien circonscrite par ces essais.

M. HALLE. - Les essais de chocs thermiques ont aussi mis en évidence des défauts au niveau des assemblages boulonnés corps-chapeau avec étanchéité par joint spiralé. La mise en cuvre de ce type de joint apparait assez incohérente : certains constructeurs préconisent un serrage métal/métal, d'autres un serrage au couple (ce qui par ailleurs procure une force d'écrasement incertaine). Quand le joint n'est pas serré métal/métal, il se trouve comprimé au-delà de sa limite élastique lors de la première montée en température ce qui est la cause certaine d'une fuite ultérieure.

Il apparait donc tout à fait nécessaire que les constructeurs de robinets se rapprochent des fabricants de joints pour définir des conditions de mise en œuvre cohérentes.

M. AFLALO. - Programmez-vous des essais d'excitation séismique?

M. HALLE. - Nous n'avons pas à EDF d'installation permettant de faire des essais de séisme. Le CEA, lui, dispose d'installations qui permettent de le faire, mais à ma connaissance, actuellement, il n'y a aucun essai réalisé sur la robinetterie.

M. AFLALO. - Les séismes doivent davantage affecter les organes de commande que le corps même du robinet.

M. HALLE. - En effet l'organe de commande est souvent situé assez loin du corps du robinet.

Le Président. - Je remercie les orateurs d'avoir bien voulu nous faire part de leurs expériences. On peut constater que l'analyse systématique des problèmes à résoudre pour assurer les différentes fonctions, pour maintenir la fiabilité, pour assurer une bonne maintenance ainsi que l'analyse des résultats obtenus aux essais ou en exploitation permettent de concevoir des appareils devant mieux satisfaire aux exigences. J'insisterai encore sur l'intérêt qu'il y a à améliorer les appareils. M. HALLE nous a parlé ce matin du nombre de robinets qu'il y a dans une tranche nucléaire de $900 \mathrm{MW}$, de leur importance, de leur incidence sur la disponibilité. Je constate, au cours des arrêts de tranches pour entretien, que le volume de l'entretien de la robinetterie représente entre 40 et $60 \%$ de l'ensemble des travaux à réaliser. Les Américains sont plus près de $60 \%$, d'après certaines informations, les Français plus ptès de 40 ou $50 \%$; mais c'est tout de même considérable, quand on pense à l'importance de ces travaux.

Je terminerai par le souhait suivant : que les efforts des concepteurs, des constructeurs, des installateurs, des responsables d'essais de contrôle, des exploitants, tels qu'ils nous ont été relatés ici cet après-midi, soient soutenus, maintenus dans une concertation permanente, parce que c'est un travail en commun qui permettra d'aboutir à des résultats concrets et à des résultats significatifs.

Il faut avoir conscience que, compte tenu de l'évolution des caractéristiques, des effets de taille, des fluides divers que l'on est amené à utiliser, cet effort sera à poursuivre encore pendan longtemps. II faudra rester vigilant et ce sera un souci de tous les instants présents et à venir.

Je vous remercie de votre attention.

La séance est levée à 17 heures. 